\title{
Sensitivity And Quantitativity In Atom Probe Tomography
}

\author{
F. Danoix ${ }^{*+}$, A. Bostel ${ }^{*}$, H. Leitner ${ }^{+}$, P. Jessner ${ }^{*}$, X. Sauvage ${ }^{*}$, M. Gouné ${ }^{* *}$, R. Danoix ${ }^{*}$ \\ * Université de Rouen - Groupe de Physique des Matériaux, UMR CNRS 6634 - Avenue de \\ l'Université - 76801 Saint Etienne du Rouvray Cedex - France \\ ${ }^{+}$Department Metallkunde und Werkstoffprüfung - Montanuniversität Leoben - Roseggerstrasse 12 \\ - A-8700 Leoben - Austria \\ ** ArcelorMittal Maizières Research SA, Voie Romaine - 57280 Maizières Les Metz - France
}

Atom probe tomography (APT) is now well recognised as a powerful instrument for nanometer scale analysis of conductive or semi-conductive materials [1]. The instrument can be regarded as an analytical microscope, generating 3D compositional maps, with a spatial resolution of a tenth of a cubic nanometer. This technique thus allows chemical information to be derived from volumes as small as $1 \times 1 \times 1 \mathrm{~nm}^{3}$, up to $100 \times 100 \times 100 \mathrm{~nm}^{3}$.

Despite the smallness of the analysed volumes, and because of the high detection efficiency (approx. $50 \%$ ) several ten millions of atoms can be collected on a single experiment. As a consequence, very small level of impurities, or allowing elements, can be revealed. In the ideal situation, levels as low as a fraction of a part per million (ppm) should easily be measured, and quantified. Unfortunately, the instrument is not background noise free, which limits its sensitivity. A rough estimate of the background noise, and therefore the 'theoretical' sensitivity of the technique are given, and compared to the measured values. This comparison shows that, for most 'light' elements, including carbon and nitrogen, a reasonable sensitivity level of approximately a few atomic part per million (at ppm) can be reached. For other elements, in particular those having masses slightly higher than the main matrix elements, the sensitivity can be deteriorated to 50 at $\mathrm{ppm}$.

In addition to sensitivity, quantitativity is a key issue for atom probe tomography as an analytical instrument. This aspect is investigated through the analysis of several 'reference' materials, most of them being selected in a wide variety of steel, including iron carbides and nitrides.

Even if the development of the latest 'wide angle' instruments [2,3] has allowed volumes of up to 100x100x100 $\mathrm{nm}^{3}$ to be investigated, these volumes are still much too small to assess the representativity of any single APT analysis. Unless specimens are prepared in a site specific approach, several similar such volumes must be analysed to secure the microstructural information obtained. To this respect, reproducibility of APT analyses must be checked, in order to make sure that composition fluctuations, when observed, arise from the material itself (from material processing to solid state phase transformations) and not from analytical aspects (from specimen preparation to possible high electric field artefacts). Some examples will be given.

References:

[1] M.K. Miller, Atom probe tomography, analysis at the atomic level, Kluwer Academic, New York, 2000

[2] see http://www.cameca.fr/html/product_atom_probe.html

[3] see http://www.imago.com/imago/html/products/LEAP3000.jsp 


\section{mass spectrum - pure iron}

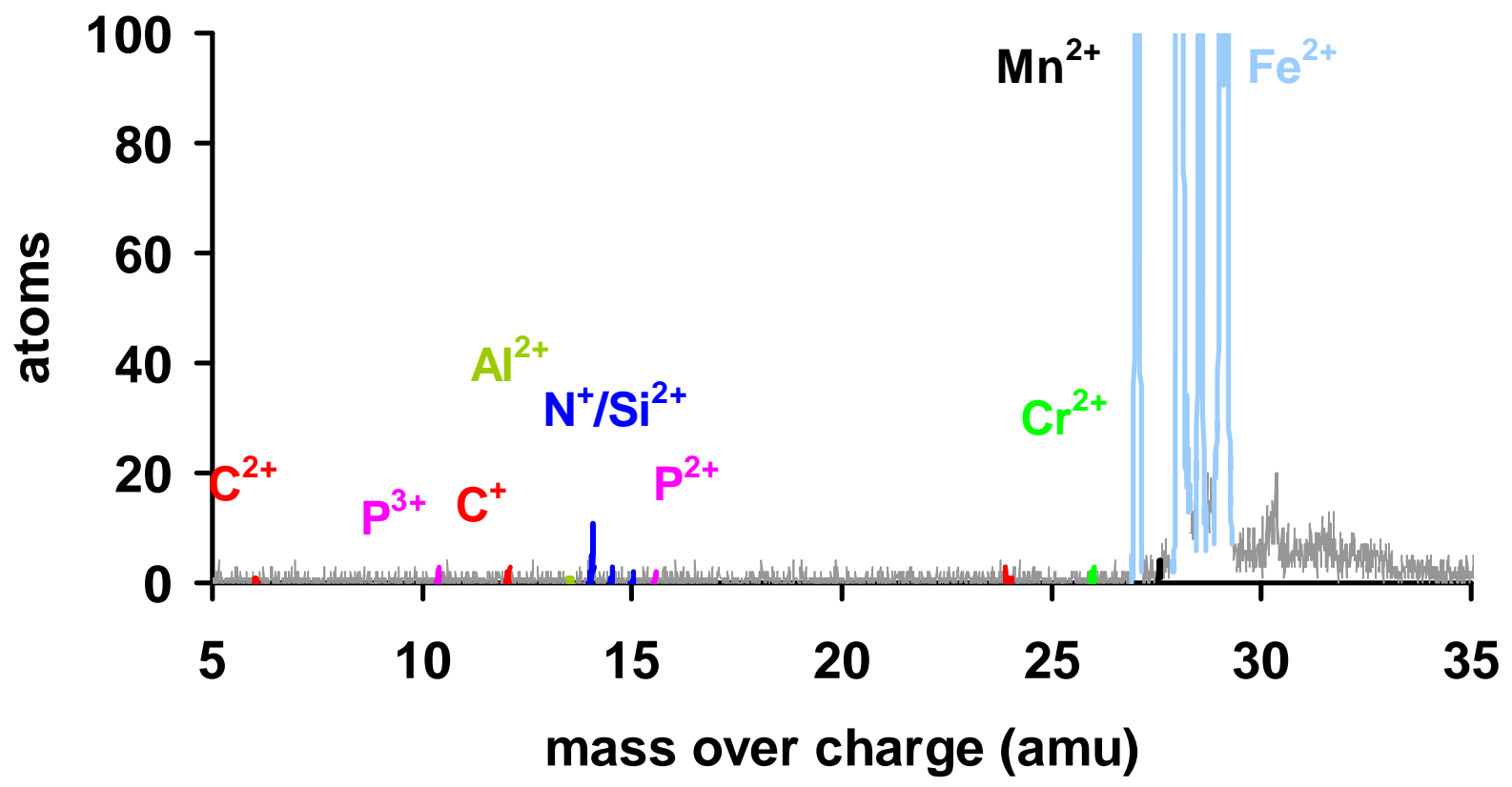

Fig. 1. Mass spectrum obtained on a high purity (99.9995\%) iron specimen. The mass over charge ratio corresponding to the common impurities are pre-defined in order the check the sensitivity of the instrument. Spectrum contains a total of 1000000 ions.

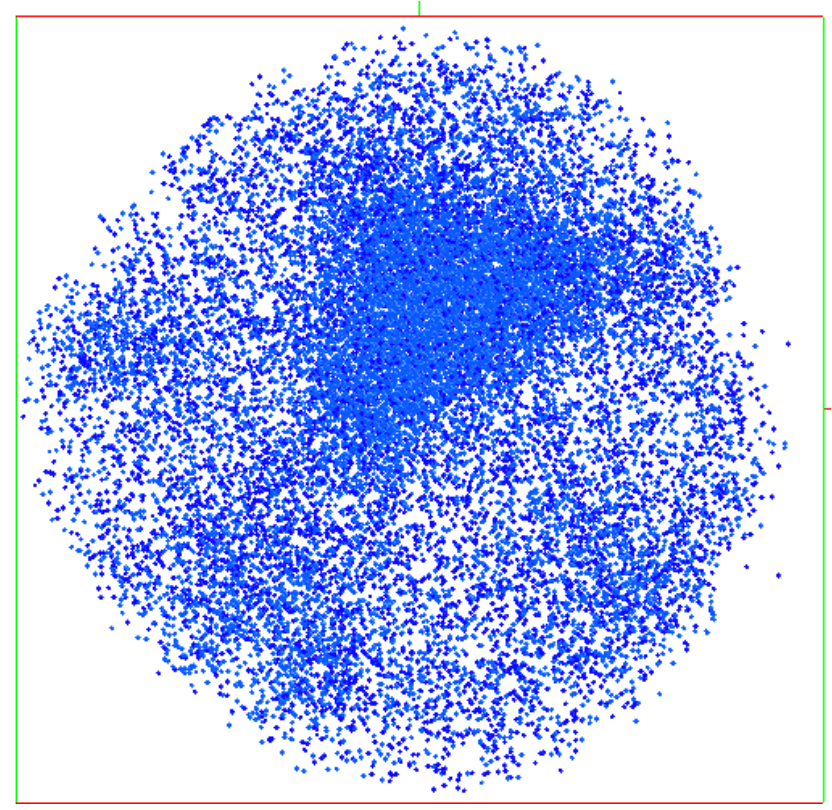

Fig. 2. Distribution of nitrogen in a model steel containing 500 atppm N. Local variations of the nitrogen composition are dependent on the crystallography of the needle tip specimen, and can lead to artefacts if not considered during data interpretation. The limiting square is $70 \times 70 \mathrm{~nm}^{2}$. 